\title{
Vascular Parkinsonism: A Clinical Study of Response to Dopaminergic Therapy and Patterns of Brain Vascular Lesions in a Group of Patients
}

Flora Zarola, $\mathrm{MD}, \mathrm{PhD} \mathrm{D}^{*}$

Neurology, Unit of Parkinson's Disease and Movement Disorders, ASL RM 6, District 2, Via Galleria di Sotto snc, Albano Laziale, Rome, Italy

\begin{abstract}
A clear-cut distinction exists between Parkinson's disease and other Parkinson-like syndromes. A commonly reported Parkinson-like syndrome is Vascular Parkinsonism (VP). The criteria to distinguish idiopathic Parkinson's Disease from VP and essential tremor (ET) are stated, like stable response to dopaminergic therapy (e.g., ex juvantibus criterion), the positivity of receptor brain tomoscintigraphy (dopamine transporter scan, Dat-scan) and nuclear magnetic resonance imaging. Recent clinical experience in our Outpatient Movement Disorders Center has revealed an apparent lack of these criteria, and the goal of this study is to spur a re-examination of the accepted criteria based on our reported experience. A group of 21 patients affected by brain vascular damage with diagnosed VP was observed in our clinic. In these patients, we detected cerebrovascular disease (CVD) which was classified into major stroke, multiple cerebrovascular lesions consisting in lacunar infarcts, leukoaraiosis with gliotic pitting, and severe signs of hypodensity of the brain white matter due to chronic hypoxia. The 21 patients were treated for longer than 24 months after our team noted a good response to dopaminergic therapy. The result was assessed based on improvement of symptoms and clinical objective examination at scheduled intervals according to the outpatients clinic's clinical protocol. We assessed the patient's unified Parkinson's disease rating scale (UPDRS) part III score at 6 months, 12 months, and 24 months. With all these criteria most patients (16 of 21) demonstrated a good stable response to dopaminergic therapy. The Fischer test was also applied to verify the significance of the results obtained as appropriate to the size of the sample analyzed. The obtained result $(p=0.0266)$ indicates that drug therapy can potentially be modified in a significant number of patients with VP because the response to dopaminergic therapy is shown not to be episodic.
\end{abstract}

\section{Keywords}

Vascular parkinsonism, Parkinson's disease, Cerebrovascular disease, Stroke, Dopaminergic therapy

\section{Introduction}

International guidelines on movement disorders and Parkinson's disease (PD) accurately describe classified extrapyramidal disorders [1]. Among the Parkinsonian Syndromes, one of the most reported is vascular Parkinsonism (VP) [2]. VP is a clinical entity distinct from PD. The criteria to distinguish idiopathic PD and VP (and ET) are both clinical and instrumental; these criteria consist of a stable response to dopaminergic therapy (ex-juvantibus criterion), the results of receptor brain tomoscintigraphy (e.g., a dopamine-transporter [DaT] scan), and nuclear magnetic resonance imaging (MRI) or computed tomography scan. In our Outpatient Movement Disorders Center, we encountered a significant discrepancy in the commonly accepted criteria for distinguishing PD from VP. This study examined the occasional clinical finding of a good response to dopaminergic therapy in patients with CVD and extrapyramidal symptoms commonly classified as VP.

\section{Materials and Methods}

We conducted a retrospective analysis of 21 VP patients suffering from extrapyramidal symptoms from January 2017 to January 2019 at our Center of Movement Disorders. The patient group included 12 men and 9 women aged 58

*Corresponding author: Dr. Flora Zarola, MD, PhD in Neurology Unit of Parkinson's Disease and Movement Disorders, ASL RM 6, District 2, Via Galleria di Sotto snc, 00041 - Albano Laziale, Rome, Italy, Tel : +39-0693273375

Accepted: February 10, 2022

Published online: February 12, 2022

Citation: Zarola F (2022) Vascular Parkinsonism: A Clinical Study of Response to Dopaminergic Therapy and Patterns of Brain Vascular Lesions in a Group of Patients. Insights Neurooncol 5(1):51-53

Copyright: (C) 2022 Zarola F. This is an open-access article distributed under the terms of the Creative Commons Attribution License, which permits unrestricted use, distribution, and reproduction in any medium, provided the original author and source are credited. 

in a Group of Patients. Insights Neurooncol 5(1):51-53

Table 1: Fisher exact test.

\begin{tabular}{|l|l|}
\hline $\begin{array}{l}\text { Patients responder to DA } \\
\text { therapy }\end{array}$ & $\begin{array}{l}\text { Patients not responder to DA } \\
\text { therapy }\end{array}$ \\
\hline 16 & 5 \\
\hline
\end{tabular}

Two-tailed: $p=0.0266^{*}$

One-tailed: $p=0.0133^{*}$

*Significant at $p<0.05 . D A=$ dopaminergic

to 93 years (mean age: 78 years). Patients were diagnosed with VP according to current clinical criteria as follows: onset of symptoms after acute cerebrovascular events (5 patients); nonlateralized onset of tremor/stiffness; the presence of the clinical syndromic scenario known as lower body parkinsonism (7 patients); in 4 other cases of acute onset after stroke, lateralized extrapyramidal symptoms coherent with the side localization of cerebral/cerebellar stroke were releved. Moreover, in 5 patients with prevalent tremor, a lower incidence of hypomimia was generally noted. Cerebrovascular disease (CVD) was classified as follows: Major stroke (ischemic or hemorrhagic); leukoaraiosis with diffuse gliotic spotting; ischemic injury load with multiple sub centimetric areas of altered signal intensity (minor stroke) in the subcortical white matter at the junctional site, with a bilateral or unilateral distribution including in some cases the basal ganglia, mesencephalic and pontine sites. In several cases, the $\mathrm{CT} / \mathrm{MRI}$ scan revealed areas of tissue necrosis on an ischemic cerebrovascular basis, which were likely from previous lacunar infarcts. In patients with a sudden onset of mostly lateral Parkinsonian symptoms, the most evident pattern of vascular damage was ischemic or hemorrhagic stroke followed by acute or subacute onset hemiParkinson, coherent with the side of the stroke. Moreover the development of CVD in the group of patients could be represented into two main descriptions: A "silent" mode with clinically "mute" lesions that rose over time to configure the pattern of multiple lesions running asymptomatic until the onset of the parkinsonian's symptoms and sudden onset with the cerebrovascular accident immediately preceding the parkinsonian's symptoms (acute or sub-acute VP onset). All patients provided written informed consent to administration of rotigotine and levodopa as dopaminergic therapy. The effectiveness of dopaminergic therapy was evaluated by means of patients subjective ameliorating perception and of clinical objective examination after the first month. These were detected from the anamnestic report and from the clinical evaluation carried out with the patient's unified Parkinson's disease rating scale (UPDRS) part III obtained from the analysis of medical records collected in a computerized system. We assessed UPDRS part III score at six months, 12 months, and 24 months and beyond. The Fisher's exact test was apllied to determine the appropriate size of the sample. The $p<0.05$ was considered to be statistically significant.

\section{Results}

Most patients (16 of 21) demonstrated a significant response to dopaminergic therapy, as evidenced by anamnestic report of improved daily tremor and/or reduced stiffness. Most important, the mean values of UPDRS part III in 16 out of 21 patients calculated at 6,12 and at 24 months were respectively $35,25,18$, therefore showing on average a good response to dopaminergic therapy with improved motor scores. The results obtained from the statistical analysis (Table 1) demonstrate a significant response to dopaminergic therapy in the patient population (Fisher Test: $p=0.0266$ ). These results show a level of efficacy of dopaminergic therapy not coherent with a random model.

\section{Discussion}

Most patients of the studied group showed clinical improvement with dopaminergic therapy even though the tests traditionally used to confirm the diagnosis of Parkinson's disease (PD) (e.g., DaT scan) were not demonstrative for PD diagnosis. Indeed the opposite situation can be observed in some cases: Sometimes patients with positive DaT scan do not respond well to dopaminergic therapy [3]. However, in clinical practice patients affected by VP have a good response to dopaminergic therapy in contrast with the above described phenomenon regarding patients with positive DaT scans who do not respond to dopaminergic drugs $[4,5]$. This justifies the broader use of dopaminergic therapy outside of routine clinical use (i.e., with dosages similar to those used in PD therapy) [6]. Low doses of levodopa or dopamine-agonists are sometimes used to treat VP [7]. A good response to dopaminergic therapy in patients who developed extrapyramidal symptoms after brain strokes have been noted previously $[2,8]$. The above reported experience gained on a large number of patients in the movement disorder clinic suggests that dopaminergic therapy can be used more extensively on patients who showed several patterns of brain vascular damage with the next onset of extrapyramidal symptons similar to PD. Our clinical experience has shown that patients stabilized and benefited from this therapy, with improvements lasting over two years. On the other hand significant comorbidity of CVD has been statistically demonstrated in patients with PD [1,2]. Therefore, there might exist a pathogenetic linkage between ischemic damage and the presence of extrapyramidal symptoms typically attributed to the original PD [9]. This suggests that acute or chronic, focal or diffuse hypoxia may play an essential role in the presynaptic damage of the nigrostriatal circuit. Moreover, vascular lesions can occur in sites independent of the side affected by the extrapyramidal symptoms in the VP patients [10], sometimes or often distant from the basal ganglia involved in the dopaminergic pathway (i.e. at cortical or subcortical level) [11]. This could explain the poor correlation often observed between the distribution pattern of vascular lesions and the clinical manifestation of extrapyramidal symptoms. In other cases a major stroke can directly involve the dopaminergic circuit like the deep arterial branches ischaemias or in some cases cerebellar strokes [12]. Therefore on the basis of these considerations it is not still possible to derive a univocal criterion for using the distribution pattern of vascular lesions as a predictive model for the onset of VP nor for the efficacy of the response to dopaminergic therapy. Therefore, extrapyramidal symptoms in CVD are not fully explained by the anatomical location of vascular lesions and damage to the nigrostriatal pathway. Biochemical parameters may be acting at a level that is not 
morphologically detectable or may involve extrapyramidal pathways in remote locations. However, in a small number of cases, ischemic or hemorrhagic stroke lesions localized in the basal ganglia sites explain the acute or subacute onset of Parkinsonian symptoms with aspects similar to idiopathic PD [13] (e.g., lateralized symptoms). The patient's susceptibility to dopaminergic therapy may vary due to an eventual disruption of the pre- and postsynaptic or axonal nervous tissue damage of the nigro-striatal pathway, depending on the ischemic insult for subjects with idiopathic PD. Probably there is a large watershed between the common diagnosed PD and the genetic forms of PD in which the mutations play a fundamental role for example inducing the synthesis of the parkin proteins and alpha-synuclein polymers. The common forms (so-called 'sporadic') probably have an extended, prevailing random and still underestimated component in vascular damage.

This study was not without limitations. Probably the study population is too small to conduct a statistically significant investigation on the possible correlation between the vascular lesion pattern (e.g., major stroke, minor stroke, multiple cerebrovascular lesions with diffuse infarcts, leukoaraiosis, ischemic injury load with variable cortical and subcortical distribution, and hemorrhagic stroke) and the degree of response to dopaminergic therapy. Previous studies reported a poor or absent response to dopaminergic therapy like in lower body parkinsonism (previously known as pseudo-bulbar syndrome): This could be due to massive nervous tissue damage produced by widespread ischemic lesions with involvement of the pre- and postsynaptic components of the nigrostriatal system [14]. An attempt to establish a correlation between the type of vascular damage and possible dopaminergic response (also useful in predicting the adoption of dopaminergic therapy) may be attempted when a much larger sample of patients is available for statistic studies. As of now, there is no predictive criterion on the efficacy of dopaminergic therapy in VP, although the statistical investigation of this study demonstrates its validity. Therefore, the availability of a larger sample would enable the establishment of predictive criteria and influence guidelines for the indication of treatment.

\section{Conclusions}

The purpose of this study was to described the occasional clinical finding of good responses to dopaminergic therapy in patients with CVD and extrapyramidal symptoms typically classified as VP. Most patients showed a good and lasting response to the dopaminergic drug, which was relatively unespected according to common clinical practice. Indeed, the clinical survey adopted in CVD patients with VP confirmed the efficacy of the dopaminergic therapy. Therefore, drug therapy behaviour can potentially be modified in many patients who have parkinsonian symptoms with signs of documented CVD because the response to dopaminergic therapy does not seem to be episodic. Our results rely on the observation of a relatively small patient population. Therefore, additional studies with large patient populations treated with dopaminergic therapy are necessary. However, PD remains at present quite distinct from VP for other respects as concerns multifactorial causes actually well known (genetic, toxic) and separated from the ischemic cause alone.

\section{Acknowledgment}

The author wishes to thank the Nurse Coordinator Francesco Pepe and Barbara Middei, all the staff, the Coordinator of Outpatient Clinic Dr Rita Bartolomei, and Director of the district 2 RM6 Dr Pierluigi Vassallo, for their organizing work.

\section{References}

1. Zarola $F$ (2017) Incidence of vascular brain damage in a population with Parkinson Disease: A clinical statistic study in comparison with a control group of patients afferent to neurological movement disorder outpatients clinic. Acta Biomed 88: 95-96.

2. Zarola F (2017) Comparison in efficacy of dopaminergic therapy between a group of: Parkinson's disease patients and a group of patients with vascular parkinsonism. Sens Res: Neurosci Modelling 1: 10.

3. Lorberboym M, Djaldetti R, Melamed E, et al. (2004) 123I-FPCIT: Spect imaging of dopamine transporters in patients with cerebrovascular disease and clinical diagnosis of vascular parkinsonism. J Nucl Med 45: 1688-1693.

4. Foltynie T, Barker R, Brayne C (2002) Vascular parkinsonism: A review of the precision and frequency of the diagnosis. Neuroepidemiology 21: 1-7.

5. Bhatia KP, Marsden CD (1994) The behavioural and motor consequences of focal lesions of the basal ganglia in man. Brain 117: 859-876.

6. Reider-Groswasser I, Bornstein NM, Korczyn AD (1995) Parkinsonism in patients with lucanar infarcts of the basal ganglia. Eur Neurol 35: 46-49.

7. Zarola F (2018) Vascular Parkinsonism sensitive to Rotigotine therapy is found in aged patients: A clinical case description. Acta Biomed 89: 99-100.

8. Zarola $F$ (2020) Incidence of brain vascular damage in a population with Parkinson's disease: Statistical comparison by age subassemblies with age homogeneous control groups. Cureus 12: e8778.

9. Zarola F (2018) Parkinson's disease is subtly distinguishable from vascular parkinsonism as shown by their variable ranges of sensitivity to dopaminergic therapy. J Clin Cell Immunol 9: 556.

10. Fénelon G, Houéto JL (1998) Vascular Parkinson syndromes: A controversial concept. Rev Neurol (Paris) 154: 291-302.

11. Rektor I, Bohnen NI, Korczyn AD, et al. (2018) An updated diagnostic approach to subtype definition of vascular parkinsonism - Recommendations from an expert working group. Parkinsonism Relat Disord 49: 9-16.

12. Wright RL, Bevins JW, Pratt D, et al. (2016) Metronome cueing of walking reduces gait variability after a cerebellar stroke. Front Neurol 7: 84.

13. Nanhoe-Mahabier W, de Laat KF, Visser JE, et al. (2009) Parkinson disease and comorbid cerebrovascular disease. Nat Rev Neurol 5: 533-541.

14. Fitz Gerald PM, Jankovic J (1989) Lower body parkinsonism: Evidence for vascular etiology. Mov Disord 4: 249-260. 